(C) 2013 IEEE. Personal use of this material is permitted. Permission from IEEE must be obtained for all other uses, in any current or future media, including reprinting/republishing this material for advertising or promotional purposes, creating new collective works, for resale or redistribution to servers or lists, or reuse of any copyrighted component of this work in other works. 


\title{
Mean Achievable Rates in Clustered Coordinated Base Station Transmission with Block Diagonalization
}

\author{
Roberto Corvaja, Member, IEEE, Juan José García Fernández, Student Member, IEEE, Ana García Armada, \\ Senior Member, IEEE
}

\begin{abstract}
We focus on the mean achievable rate per user of the coordinated base station downlink transmission in a clustered cellular environment, with transmit power constraints at the base stations. Block Diagonalization is employed within the cluster to remove interference among users while the interference from other clusters remains. The average achievable rate per user is evaluated considering the effects of the propagation channel and the interference and a theoretical framework is presented to provide its analytical expression, validated by simulation results with different power allocation schemes. As an application, the number of cells of the cluster that maximizes the mean achievable rate per user is investigated. It can be seen that in most of the cases a reduced cluster size, close to seven cells, guarantees a rate very close to the maximum achievable.
\end{abstract}

Index Terms-Coordinated base stations, clustering, block diagonalization, network MIMO.

\section{INTRODUCTION}

Space-Division Multiplexing (SDM) based on Multiple Input-Multiple Output (MIMO) techniques has been adopted in recent wireless standards, in order to improve the data rates. However, the gains of MIMO processing in cellular networks are hampered by the interference that characterizes these environments. The classical approach to cope with interference in cellular systems has been frequency reuse with the inherent loss in spectral efficiency. If we would like to achieve spectrally-efficient communications, it is mandatory that all cells/sectors operate on the same frequency channel, what is denoted as universal frequency reuse (UFR). With this motivation, some new techniques have emerged to manage interference in cellular systems with UFR, by introducing coordination among the base stations in the downlink, which are known as network MIMO or coordinated base station transmission (CBST) [1]. In [2] the other-cell interference (OCI) is considered when designing the transmission for a multi-user MIMO downlink. In [3] the authors analyze several approaches for overcoming interference in SDM MIMO cellular networks. If the interference is known by the transmitters, cooperative encoding among base stations using Dirty Paper Coding (DPC) can suppress OCI [4]. In [1] several strategies

This work is partly funded by projects "GRE3N": TEC2011-29006-C03-03 and "COMONSENS": CSD2008-00010.

Roberto Corvaja is with the Department of Information Engineering, University of Padova, Padova, Italy. (e-mail: corvaja@dei.unipd.it).

Juan José García Fernández and Ana García Armada are with the Department of Signal Theory and Communications, Universidad Carlos III de Madrid, 28911 Leganés, Spain. (e-mail: \{jjgarcia, agarcia\}@tsc.uc3m.es). are proposed to perform CBST. Interference is eliminated by jointly and coherently coordinating the transmission from the base stations in the network, assuming that base stations know all downlink signals. Besides DPC, they propose a zero-forcing (ZF) scheme that, although suboptimal, does not involve the complexity of DPC. The capacity of MIMO benefits from CBST not only because of the rise of the operating signal to interference plus noise ratio (SINR) point, but also from the better rank condition of the joint channel matrix resulting from non-collocated base stations [5]. Similarly to multi-user MIMO, Block Diagonalization (BD) [6], [7] may be applied for CBST as a good compromise between complexity and performance. In [8] BD is applied in a multicell scenario in combination with the OCI reduction scheme of [2]. Alternatively in [9] a Singular Value Decomposition (SVD) approach is proposed that simplifies the channel estimation requirements at the expense of a performance degradation. Other linear schemes based on minimizing the mean squared error have also been proposed [10],[11].

The main drawback of all these systems is that they require channel state information (CSI) and transmit data to be simultaneously known to all cooperating base stations, with the cost of higher backhaul capability and increased signal overhead, which could even question the effectiveness of coordination [12],[13]. In particular in [12] it is shown that coordination reaches a saturation region where no increase of SNR can lead to a better performance. An important open question is whether this saturation occurs at operational SNR values. Some recent approaches have been proposed to avoid CSI and data sharing. Non coherent joint processing [14] does not require cell-to-cell CSI exchange at the expense of higher processing cost at the receivers with successive interference cancellation. In [15] the case of distributed cooperation is analyzed where each base station has only local CSI. As a practical alternative, we focus here on clustered coordination, where only a limited number of base stations can cooperate in order for the overhead to be affordable. Base stations are grouped into cooperation clusters and only the base stations of each cluster exchange information and jointly process signals. In [16] clustered coordination is analyzed, where clusters are of limited size. This has been shown to be a good trade-off between performance and overhead. Even higher performance gains can be attained if the clusters are formed dynamically [17], [18] for example by user-centric approaches [19].

Note however that none of these works analyzed the effect 
of the size of the clusters of cooperating base stations and the effect of interference coming from outside the clusters. In fact a reduction of the number of cooperating base station reduces at the same time the complexity, the overhead and the difficulty to obtain the CSI. Here we focus on this reduction of the cooperating base stations, by grouping them into fixed non-overlapping clusters, and we analyze the effect of the interference from outside the cluster. In terms of complexity non-overlapping and fixed clusters reduce the complexity, which is understood not as the mere coordination signalling overhead, but also as the signalling to manage the coordinated base stations. In previous works [8] shows by simulation that a small cluster size is sufficient to obtain most of the sum rate benefits from clustered coordination. Equivalent conclusions are obtained in [20], that also evaluated by simulation the throughput performance of MIMO techniques serving a dense population of stationary users with scheduled packet data. The authors of [21] developed analytical expressions for pure ZF in a 2- or 3-cell network with base stations transmitting at full power and each of the mobile stations equipped with just one receive antenna. Note however that clustering is not considered, since they assume that two or three cells constitute the whole network. In [22] the resource allocation problem is addressed for a large network with 3 -sector base stations. The system is statically divided into a number of disjoint clusters of 3 sectors and zero forcing is used to cancel interference within each cluster. Two cooperative frequency reuse approaches are designed to mitigate the inter-cluster interference.

In this work we focus on clustered BD-based CBST with per base station power constraints and present an analytical evaluation of the average user rates that can be achieved with different cluster sizes. Although the increased signalling required for the exchange of the channel state information is normally not considered [14]-[20], since the increased traffic on the control plane can have a limited impact on the useful traffic on the user plane, here we present some results also considering some signalling overhead, as in [12]. For this environment, other different approaches, such as dynamic or usercentric clusters, and several transmission techniques, besides MMSE or BD, have been proposed and studied [23]. Here we consider fixed non-overlapping clusters since they reduce the management complexity and $\mathrm{BD}$, since its implementation, contrary to MMSE, does not require any knowledge of out-ofcluster interference to be effective and any coordination can be limited to each cluster. Moreover, for a similar scenario such as the broadcast MIMO channel, BD proves to be quite close to optimum [24],[25]. Based on this experience on the broadcast channel, several authors, e.g. [2],[8],[28], proposed the use of $\mathrm{BD}$ also for the cooperative downlink scenario, being the main difference with respect to the broadcast channel the power constraint that is distributed among different base stations.

We derive an analytical expression for the mean achievable rate per user with $\mathrm{BD}$, which accounts for the propagation characteristics, the antenna configuration, the thermal noise level and the cluster size. We show that the power needed to coordinate the users and to reduce the interference within the cluster determines a lower power available for each parallel stream in the spatial multiplexing scheme and a trade-off occurs between the noise level and the interference coming from outside the cluster, that depends on the cluster size. We can see that in a wide range of operational conditions a reduced cluster size around seven cells gives a performance quite close to the maximum achievable. The conclusions drawn are specific to the particular choice of BD, although some general design guidelines can be obtained, confirmed by some more general studies on the subject [12].

Notation: Boldface symbols will be used for matrices and vectors. Superscripts ${ }^{T}$ and ${ }^{H}$ denote the transpose and the Hermitian transpose of a matrix, respectively.

\section{System And Cluster Model}

We target a coordinated transmission downlink scenario where cells of radius $R_{\text {cell }}$ are grouped into clusters. Clusters are composed of $M$ base stations (BS) that coordinate their transmission serving a total of $N$ users in each cluster. An example is depicted in Fig. 2 for $M=7$, showing the cell radius $R_{\text {cell }}$, i.e. the radius of the circle which circumscribes the cell. We consider non-overlapping clusters, that is, each cell belongs to just one cluster. Although overlapping usercentric clusters could provide a better performance [19], this approach gives rise to a dramatic increase of the management complexity. The clusters are defined by the network planner and are kept fixed, grouping the BSs according to a distance criterion. In our setup the cluster members are all the base stations inside the cluster, in other words, scheduling or adaptive selection of the active base stations are not addressed here, but they could be considered as a special case of optimization of the power allocation scheme.

The BSs have a maximum available power $P_{\max }$ and are equipped with $t$ transmit antennas, while the user terminals have $r$ receive antennas. The signals coming from the BSs in a cluster cause interference to other clusters since there is no coordination among clusters. The size of the cluster $M$ is then one of the key parameters that we consider in the analysis. Fig. 1 presents an example of the system showing the transmission within a single cluster with $M=3$ cells, $N=2$ active users (denoted by user 1 and user 2), $t=3$ transmit antennas and $r=1$ receive antenna.

\section{A. Channel model}

The propagation inside a cluster is modelled by a $\mathrm{Nr} \times \mathrm{Mt}$ channel matrix $\mathbf{H}$ whose elements are the fading coefficients from any transmit antenna of each BS to any receive antenna of each user. Then the total channel can be written as $\mathbf{H}=\left[\mathbf{H}_{1}^{\mathrm{T}} \mathbf{H}_{2}^{\mathrm{T}} \ldots \mathbf{H}_{N}^{\mathrm{T}}\right]^{\mathrm{T}}$, where $\mathbf{H}_{i}, \quad i=1 \ldots N$ is $r \times M t$ channel matrix seen by user $i$, which contains $M$ sub-matrices $\mathbf{H}_{i j}$ of size $r \times t$ representing the channel from the $\mathrm{BS} j$. An example is shown in Fig. 1 where the contributions $\mathbf{H}_{i j}$ are shown for the user 2. In terms of propagation, the channel matrix is usually decomposed into a spatial correlation at the transmitter, a fading matrix and a spatial correlation at the receiver. In the following we consider spatially uncorrelated antennas at both transmitter and receiver and Rayleigh fading, as in [1], so that he entries of the matrix $\mathbf{H}_{i}$ are iid complex Gaussian random 


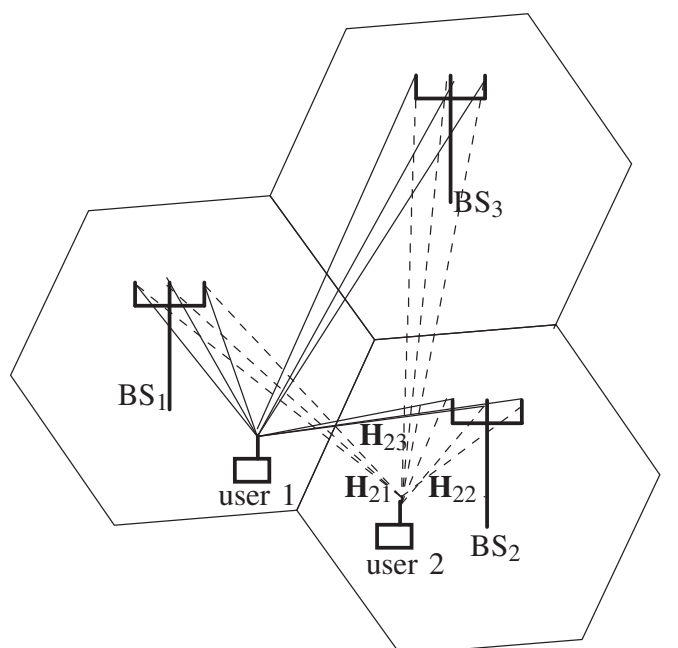

Fig. 1. Model of a cluster with $M=3$ BSs and $N=2$ active users. The contributions $\mathbf{H}_{2 j}, \quad j=1,2,3$ from each BS to the overall channel $\mathbf{H}_{2}$ of the user 2 are shown.

variables with zero mean and a variance which represents the power path loss between base station $j$ and user $i$. The path loss varies according to an exponential power decay with exponent $\gamma$, namely $u_{i j}^{-\gamma}$, as a function of the distance $u_{i j}$ from user $i$ to $\mathrm{BS} j$. The received signal is thus

$$
\mathbf{y}=\mathbf{H x}+\mathbf{n}
$$

where $\mathbf{y}$ is the received $N r \times 1$ signal vector, $\mathbf{x}$ the $M t \times 1$ signal vector transmitted from all the coordinated BSs and $\mathbf{n}$ is the $N r \times 1$ vector of i.i.d complex Gaussian noise entries with zero-mean and variance $\sigma_{n}^{2}$.

Since the received power varies according to the user location, the amount of noise and the value $\rho$ of the signal to noise ratio (SNR) are defined with reference to the power received at the cell border, as done also in e.g. [8], namely

$$
\rho=\frac{P_{\max } R_{c e l l}^{-\gamma}}{\sigma_{n}^{2}} .
$$

\section{B. Interference model}

In Fig. 2 we show an example of cluster layout, where three complete clusters each consisting of seven cells are presented, together with other cells belonging to other clusters, not completely shown. The user $i$, at the distance $u_{i}$ from its serving BS, is affected from interference coming from the neighbor clusters. The interfering cells belonging to the first tier are located at a distance $D_{1}$ (or greater) from $\mathrm{BS}_{i}$. Similarly for the user $j$ at distance $u_{j}$ from $\mathrm{BS}_{j}$, the first tier of interfering cells is at a distance $D_{2}$ from $\mathrm{BS}_{j}$. Due to the cellular geometry, for a cluster size up to 18 , only these two possibilities exist: the closest interfering cell is located at either $D_{1}$ or $D_{2}$ from the serving BS of each user. According to a uniform distribution of the users over each cell, the distance $u_{i}$ of user $i$ from the nearest BS has a probability density function

$$
f_{u_{i}}\left(u_{i}\right)=2 u_{i} / R_{\text {cell }}^{2},
$$

which approximates the hexagonal cell by a circular one with the same radius (see Fig. 2). In the following the subscript $i$

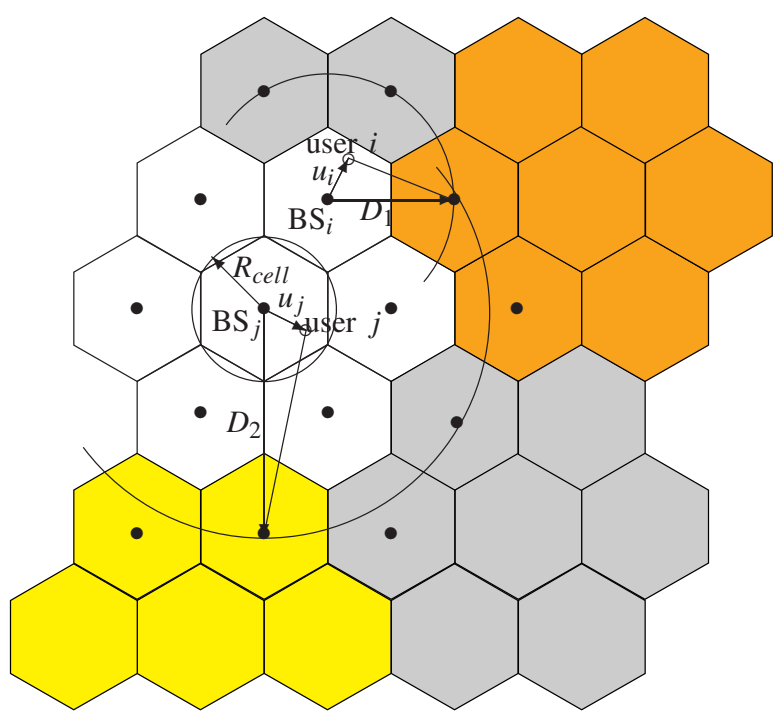

Fig. 2. System layout with clusters of seven cells of radius $R_{\text {cell }}$ (the radius of the circle circumscribing the cell) with an example of two users, with the distances $D_{1}$ and $D_{2}$, respectively, from their BS to the interfering BSs.

is skipped. The interference power is given by

$$
I_{i}(u)=\sum_{m=1}^{M_{\text {interf }}} P_{\max }\left(d_{i m}\right)^{-\gamma},
$$

where $M_{\text {interf }}$ is the number of interfering stations and $d_{i m}$ is the distance between user $i$ and base $m$ (belonging to the external clusters). To account for the interfering power, we introduce an equivalent model, providing the same amount of interference, but where all the interfering BSs for each user are located at the same distance, namely the minimum distance, either $D_{1}=\sqrt{3} R_{\text {cell }}$ or $D_{2}=3 R_{\text {cell }}$ (see Fig. 2). In this way some BSs are actually considered closer than what they really are, and this effect is accounted for with an equivalent number of interfering base stations $M_{e q, i}$. For example, all the interfering stations are considered at $D_{1}-u_{i}$ from user $i$, while for user $j$ at $D_{2}-u_{j}$, and so on. The distance according to the model above is given by $d_{i j}=D_{i}-u$ for all the stations of the adjacent clusters, so that the interference is represented by a term

$$
I_{i}(u)=P_{\max } M_{e q, i}\left(D_{i}-u\right)^{-\gamma},
$$

where $D_{i}$ can be either $D_{1}$ or $D_{2}$ (see Fig. 2) and we assume that the base stations transmit at the maximum power. This approach is similar to what is considered in [26], where a continuous of interference is assumed, so that all the interfering BSs are spread over the whole plane but at the same distance from the user, with a suitable probability density function (pdf) of the distance.

The real and equivalent models produce the same total interference power for each user if $M_{e q, i}$ is adequately selected. In order to determine $M_{e q, i}$ we consider the interference coming from the first tier of neighbor cells, so that for different cluster configurations we have a different number of interfering base stations for each cell. This is clear in Fig. 3 where a cluster of $M=4$ cells is shown, in which users in cells 1 and 3 experience an interference coming from $M_{i n t, 1}=M_{i n t, 3}=4$ neighbor cells, while cells 2 and 4 receive interference from 
$M_{i n t, 2}=M_{i n t, 4}=3$ cells. For example, if we consider $M=1$,

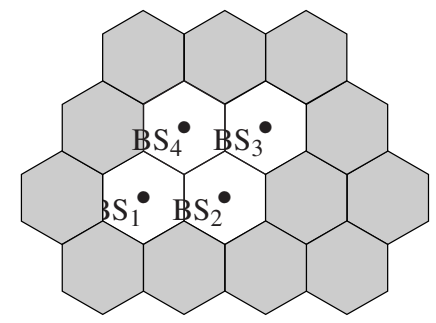

Fig. 3. Cluster with $M=4$ cells and neighbor interfering cells.

we have that the user $i=1$ in the cell is affected by an interference power $I_{1}$ which comes from all the $M_{\text {int }, 1}=6$ cells belonging to the first tier. For these, we can consider that half of them $\left(M_{i n t, 1} / 2=3\right)$ are located at $\left(D_{1}-u\right)$ and half of them at $\left(D_{1}+u\right)$. Accordingly, we have

$$
\begin{aligned}
& I_{1}=P_{\max }\left[\frac{3}{\left(D_{1}+u\right)^{\gamma}}+\frac{3}{\left(D_{1}-u\right)^{\gamma}}\right]=P_{\max } M_{e q, 1} \frac{1}{\left(D_{1}-u\right)^{\gamma}} \\
& M_{e q, 1}=3\left[1+\frac{\left(D_{1}-u\right)^{\gamma}}{\left(D_{1}+u\right)^{\gamma}}\right] .
\end{aligned}
$$

In general, if we consider for user $i$ that $M_{\text {int }, i}$ interfering BSs belong to the first tier at distance $D_{i}$, the number of equivalent interfering station is given by

$$
M_{e q, i} \approx \frac{M_{\text {int }, i}}{2}\left[1+\left(\frac{D_{i}-u}{D_{i}+u}\right)^{\gamma}\right]
$$

In order to evaluate the value (8) we can set the distance $u$ to the average distance of the user within the cell. A similar approach is used also in [27] to characterize the statistics of the interference in a multi-cell scenario. With this average value, derived from (3), namely $E[u]=2 R_{\text {cell }} / 3$, we have

$$
M_{\text {eq }, i} \approx \frac{M_{\text {int }, i}}{2}\left[1+\left(\frac{D_{i}-2 R_{\text {cell }} / 3}{D_{i}+2 R_{\text {cell }} / 3}\right)^{\gamma}\right] \text {. }
$$

We see in the comparison between the analytical results and the simulations of Section $\mathrm{V}$, that this approximation is rather good.

\section{BLOCK DIAGONALIZATION}

From (1) we express the received signal as

$$
\mathbf{y}=\mathbf{H} \mathbf{x}+\mathbf{n}=\mathbf{H W b}+\mathbf{n},
$$

in which the signal vector $\mathbf{x}$ within a cluster is obtained by applying a precoding (or beamforming) matrix, that is $\mathbf{x}=\mathbf{W b}$, with $\mathbf{b}=\left[b_{11}, \ldots, b_{1 r}, \ldots, b_{N r}\right]^{\mathrm{T}}, b_{i \ell}$ represents the symbol of the $\ell$-th data stream of user $i$, transmitted with power $P_{i \ell}$ and $\mathbf{W}=\left[\mathbf{w}_{11}, \ldots, \mathbf{w}_{1 r}, \ldots, \mathbf{w}_{N r}\right]$ is the beamforming matrix, where $\mathbf{w}_{i \ell}=\left[w_{i \ell}^{11}, \ldots, w_{i \ell}^{1 t}, \ldots, w_{i \ell}^{k l}, \ldots, w_{i \ell}^{M t}\right]^{\mathrm{T}}$ are the precoding vectors for the $\ell$-th data stream of the $i$-th user. The beamforming matrix $\mathbf{W}$ is obtained under a $\mathrm{BD}$ criteria as in $[1],[28]$, to guarantee that

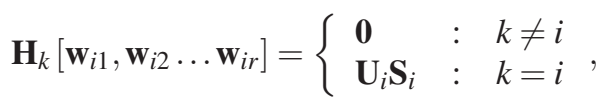

where $\mathbf{U}_{i}$ is unitary and $\mathbf{S}_{i}=\operatorname{diag}\left\{\lambda_{i 1}^{1 / 2}, \lambda_{i 2}^{1 / 2}, \ldots, \lambda_{i r}^{1 / 2}\right\}$. The $\lambda_{i \ell}^{1 / 2}$ are obtained from a singular value decomposition of the interfering channels according to the procedure explained in [28]. Then, the received signal can be expressed as

$$
\mathbf{y}=\left[\begin{array}{cccc}
\mathbf{U}_{1} \mathbf{S}_{1} & \mathbf{0} & \ldots & \mathbf{0} \\
\mathbf{0} & \mathbf{U}_{2} \mathbf{S}_{2} & \ldots & \mathbf{0} \\
\vdots & \vdots & \ddots & \vdots \\
\mathbf{0} & \mathbf{0} & \ldots & \mathbf{U}_{N} \mathbf{S}_{N}
\end{array}\right] \mathbf{b}+\mathbf{n} .
$$

Each user independently rotates the received signal and decouples the different streams

$$
\widetilde{\mathbf{y}}=\left[\begin{array}{cccc}
\mathbf{U}_{1}^{H} & \mathbf{0} & \ldots & \mathbf{0} \\
\mathbf{0} & \mathbf{U}_{2}^{H} & \ldots & \mathbf{0} \\
\vdots & \vdots & \ddots & \vdots \\
\mathbf{0} & \mathbf{0} & \ldots & \mathbf{U}_{N}^{H}
\end{array}\right] \mathbf{y}=\left[\begin{array}{c}
\lambda_{11}^{1 / 2} b_{11} \\
\vdots \\
\lambda_{1 r}^{1 / 2} b_{1 r} \\
\vdots \\
\lambda_{N r}^{1 / 2} b_{N r}
\end{array}\right]+\widetilde{\mathbf{n}}
$$

where the noise $\widetilde{\mathbf{n}}$ remains white with the same covariance because of the unitary transformation. BD is possible in this scenario if the condition $M t \geq N r$ is satisfied. This approach assumes the knowledge of the CSI for the coordinated cells, i.e. inside the clusters. The effect of imperfect channel knowledge has been considered in [28], where we showed the limits, in terms of channel estimation error, required to achieve a mean achievable rate close to the ideal one obtained with perfect CSI.

\section{ANAlysis OF THE RATE}

With respect to the ideal case of a single cluster, where interference among the users is cancelled by BD, in a multicluster environment we have to consider the effect of the interference coming from the cells outside the cluster. Hence, the achievable rate of user $i$ is

$$
R_{i}=\sum_{\ell=1}^{r} \log _{2}\left(1+\lambda_{i \ell} \frac{P_{i \ell}}{\sigma_{n}^{2}+I_{i}}\right) .
$$

where the parameter $I_{i}$ represents the average power of the total interference contributions received by each antenna of user $i$ from the interfering base stations. In order to evaluate (14) we notice that the rate depends on the distance $u$ of each user's equipment from the center of its cell. Introducing the pdf of distance given by (3) and making explicit the dependence of the parameters on the distance $u$, the expectation of (14) becomes

$$
\overline{R_{i}}=\int_{0}^{R_{\text {cell }}} \sum_{\ell=1}^{r} \log _{2}\left(1+\lambda_{i \ell}(u) \frac{P_{i \ell}}{\sigma_{n}^{2}+I_{i}(u)}\right) \frac{2 u}{R_{\text {cell }}^{2}} d u .
$$

In (15) we have three parameters determining the overall rate, namely:

- The interference $I_{i}(u)$, coming from outside the cluster.

- The effect of the channel fading and of the path loss, represented by the term $\lambda_{i \ell}(u)$.

- The power $P_{i \ell}$ assigned to the $\ell$-th stream of user $i$.

In the following, the characterization of each parameter will be approached separately. 


\section{A. Interference}

As described in Section II-B the contribution of interference $I_{i}(u)$ on each receive antenna of user $i$ coming from the cells outside the cluster, can be considered as generated by an equivalent number of BSs, located all at the distance $D_{i}-u$ from the user, where $D_{i}$ will be either equal to $D_{1}$ or to $D_{2}$, for users in clusters whose size is up to 18 cells. The interfering distance $D_{i}$ is then normalized to the cell radius $R_{\text {cell }}$, setting $D_{i}=d_{i} R_{\text {cell }}$. In (14) we assume that the interference can be treated as Gaussian noise, so that its statistical power is required. ${ }^{1}$

\section{B. Fading effect}

The terms $\lambda_{i \ell}$ are the squared diagonal values of the matrix $\mathbf{S}_{i}$. This matrix is obtained as we can see from (11)-(12) by the combination of the channels $\mathbf{H}_{i}$ containing all the fading coefficients, with the elements of a unit-norm matrix $\mathbf{W}$. We notice that the channel matrix $\mathbf{H}_{i}$ is composed of the submatrices $\mathbf{H}_{i j}$, where the fading elements of $\mathbf{H}_{i i}$ have a power path loss $u_{i i}^{-\gamma}$, while the elements of $\mathbf{H}_{i j}$ are independent of $\mathbf{H}_{i i}$ with path loss $u_{i j}^{-\gamma}$, where $u_{i j}$ is the distance of the user $i$ from the base station $j$. Then, by separating the path loss corresponding to the distance from the nearest base station, namely $u_{i i}$, we can write $\lambda_{i \ell}=\mu_{i \ell} u_{i i}^{-\gamma}$ where $\mu_{i \ell}$ are the elements of the diagonal matrix $u_{i i}^{-\gamma} \mathbf{S}_{\ell} \mathbf{S}_{\ell}^{H}$. We note that these diagonal elements are the singular values of the matrix $u_{i i}^{-\gamma} \mathbf{H}_{i} \mathbf{W} \mathbf{W}^{H} \mathbf{H}_{i}^{H}$ where $\mathbf{H}_{i}$ has Gaussian entries and $\mathbf{W}$ is unitary. These are the eigenvalues of a Wishart matrix in the case $M t=N r$, while in the general case with $M t \geq N r$, this matrix can be approximated by a Wishart matrix. ${ }^{2}$ The joint pdf of these $\mu_{i \ell}$ for a Wishart matrix can be obtained when the columns of the corresponding Gaussian matrix have identity covariance matrix $\Sigma=\mathbf{I}$. The joint pdf is given by [29]

$$
f\left(\mu_{i 1}, \ldots, \mu_{i r}\right)=e^{-\sum_{n=1}^{r} \mu_{i n}} \prod_{n=1}^{r} \frac{1}{[(r-n) !]^{2}} \prod_{m>n}^{r}\left(\mu_{i m}-\mu_{i n}\right)^{2} .
$$

However, in the rate evaluation the complete joint pdf is not needed, since only the sum $\sum_{i=1}^{N} \sum_{\ell=1}^{r} \log \left(\mu_{i \ell}\right)$ is required, which represents the expectation of the logarithm of the determinant, for which results are available also for a generic covariance matrix $\Sigma \neq \mathbf{I}$, given by [29]

$$
E\left[\sum_{i=1}^{N} \sum_{j=1}^{r} \log \left(\mu_{i \ell}\right)\right]=\sum_{k=0}^{N r-1} \psi(N r-k)+\log (|\Sigma|)
$$

where $\psi(\cdot)$ is the Euler's digamma function [30]. The columns of the matrix $u_{i i}^{-\gamma / 2} \mathbf{H}_{i} \mathbf{W}$ have covariance matrix

$$
\Sigma=\mathbf{I}\left[1+\sum_{j=1}^{M}\left(\frac{u_{i i}}{D_{j i}}\right)^{\gamma}\right]
$$

\footnotetext{
${ }^{1}$ In simulations we found that on average at least 25 out-of-cluster cells contribute with interference to a given user with a power greater than the received useful power at the cell edge. This number corresponds to a scenario with cell radius of $1400 \mathrm{~m}$ and propagation exponent 3.8.

${ }^{2}$ By simulation we derive that the mean of the values $\mu_{i \ell}$ is the same and the maximum difference between the two CDFs is less than $10 \%$.
}

We can define the parameter $F$

$$
F=1+\sum_{j=1}^{M}\left(\frac{u_{i i}}{D_{j i}}\right)^{\gamma}
$$

which can be considered as a cluster gain. Its value again can be obtained considering the user at the average distance $u_{i i}=\frac{2}{3} R_{c e l l}$ and the base stations at the distances $D_{1}$ or $D_{2}$

$$
F=\left\{\begin{array}{lll}
1+\frac{M-1}{2}\left[\left(\frac{\frac{2}{3} R_{\text {cell }}}{D_{1}-\frac{2}{3} R_{\text {cell }}}\right)^{\gamma}+\left(\frac{\frac{2}{3} R_{\text {cell }}}{D_{1}+\frac{2}{3} R_{\text {cell }}}\right)^{\gamma}\right] & M \leq 7 \\
3\left[\left(\frac{\frac{2}{3} R_{\text {cell }}}{D_{1}-\frac{2}{3} R_{\text {cell }}}\right)^{\gamma}+\left(\frac{\frac{2}{3} R_{\text {cell }}}{D_{1}+\frac{2}{3} R_{\text {cell }}}\right)^{\gamma}\right] & \\
+\frac{M-7}{2}\left[\left(\frac{\frac{2}{3} R_{\text {cell }}}{D_{2}-\frac{2}{3} R_{\text {cell }}}\right)^{\gamma}+\left(\frac{\frac{2}{3} R_{\text {cell }}}{D_{2}+\frac{2}{3} R_{\text {cell }}}\right)^{\gamma}\right] & &
\end{array}\right.
$$

In Fig. 4 we consider a fixed distance $u=\frac{2}{3} R_{\text {cell }}$ and we compare the sum of the log values obtained by simulation and by expressions (17)-(20), for the case $r=t=2$. The effect of

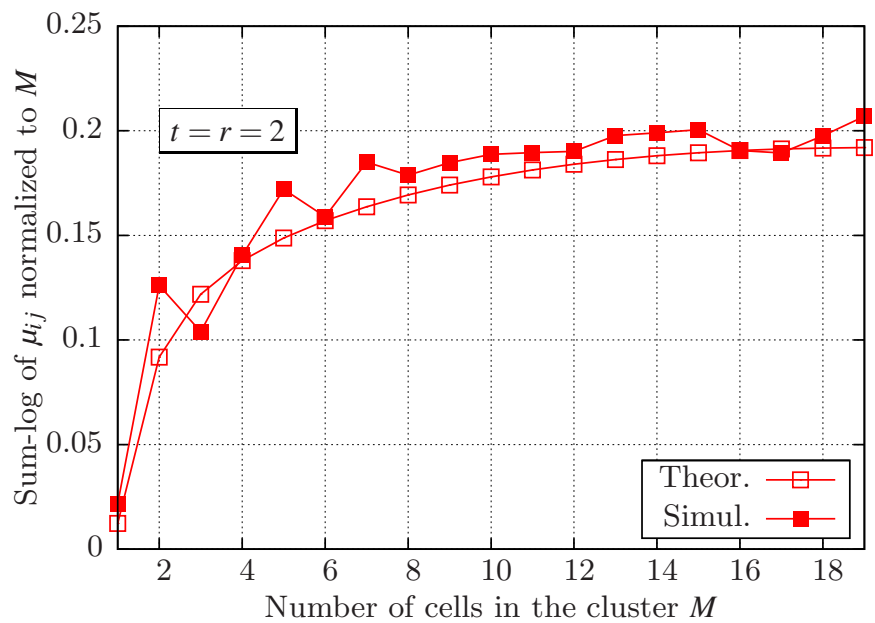

Fig. 4. Sum-log of the terms $\mu_{i j}$ : Comparison between simulation and (17) for $r=t=2$.

increasing the cluster size is to reduce the contribution of the farthest base stations, which becomes negligible due to the path-loss. Therefore we can see a saturation of the trace and of the sum-log of the values $\mu_{i \ell}$, by increasing the size of the cluster $M$. Notice that in the evaluation of the mean achievable rate we apply a factor $1 / N$ in order to evaluate the average rate per user, taking into consideration that in a bigger cluster we have also more users. This factor determines a decrease of the trace with respect to $N$ and correspondingly, of the average value of $\mu_{i \ell}$. Thus a decrease occurs in the mean achievable rate per user for large values of $N$, as we will see in Section V.

\section{Power allocation}

Under the BD strategy the transmission within each cluster is equivalent to a set of parallel non-interfering channels. Then the power must be allocated to maximize some quality of service parameters, such as the sum rate (or a weighted sum of the rates), for the users of each cluster. This objective is subject to a maximum transmission power available at each 
base station $P_{\text {max }}$, namely

$$
\sum_{l=1}^{t} \sum_{i=1}^{N} \sum_{\ell=1}^{r} P_{i \ell}\left|w_{i \ell}^{k l}\right|^{2} \leq P_{\max },
$$

for each $\mathrm{BS} j=1, \ldots, M$. The rate maximization problem has been tackled in several works, e.g. [2],[28] and solutions range from the simplest uniform power approach to optimal allocation, whose derivation requires the cumbersome numerical solution of the convex optimization problem. A power allocation solution, which resembles the well known waterfilling scheme and performs very close to the optimum, has been presented in [28]. In the following a theoretical framework is derived for the uniform power allocation scheme, which is the simplest. In any case, here we are not interested in the problem of power allocation, since it can be solved separately and the actual power, if different form the uniform, could be inserted in the analytical expression that we develop. An example, with a different power allocation, will be presented in the results.

With a uniform power allocation, a common average value $P_{0}$ replaces $P_{i \ell}$ in (15), representing the average transmitted power from the coordinated BSs to each receive antenna of user $i$. This value of power $P_{0}$, allocated to each of the $r$ parallel streams of user $i$, varies according to the number of BSs in the cluster, decreasing for a larger size of the cluster, since a fraction of the overall available power is spent for the coordination, to null the interference.

If we substitute a common value $P_{0}$ for each $i=1, \ldots, N$ and $\ell=1, \ldots, r$ the condition (21) is limited by the BS for which the following sum is maximum

$$
\sum_{l=1}^{t}\left|w_{i \ell}^{k l}\right|^{2}
$$

By using a Gaussian approximation of the coefficients $w_{i \ell}^{k l}, P_{0}$ is then related to the reciprocal value of the maximum of $M$ random variables

$$
P_{0}=\frac{P_{\max }}{E[\chi]} \quad \chi=\max \left\{\chi_{1}, \ldots, \chi_{M}\right\}
$$

with $\chi_{i}$ chi-squared with $t$ degrees of freedom. The probability distribution function of $\chi$ is

$$
F_{\chi}(x)=P(t, x)^{M}
$$

being $P(\cdot, \cdot)$ the regularized Gamma function. The mean value is derived from the probability distribution function as

$$
E[\chi]=\int_{0}^{+\infty}\left(1-F_{\chi}(x)\right) d x
$$

and can be evaluated using the bounds

$$
\left(1-e^{-\alpha x}\right)^{a} \leq P(a, x) \leq\left(1-e^{-\beta x}\right)^{a}
$$

with

$$
\begin{aligned}
\alpha & =\left\{\begin{array}{ll}
1 & 0<a<1 \\
d_{a} & a>1
\end{array} \quad \beta= \begin{cases}d_{a} & 0<a<1 \\
1 & a>1\end{cases} \right. \\
d_{a} & =(\Gamma(a+1))^{-\frac{1}{a}}
\end{aligned}
$$

where $\Gamma(\cdot)$ is the Gamma function. Then $E[\chi]$ is bounded by

$$
\frac{1}{\beta}\left[\psi(M t+1)+\gamma_{0}\right] \leq E[\chi] \leq \frac{1}{\alpha}\left[\psi(M t+1)+\gamma_{0}\right]
$$

with $\psi$ the digamma function and $\gamma_{0}$ the Euler constant. In terms of $P_{0}$, we have

$$
P_{\max } \frac{\Gamma(t+1)^{-1 / t}}{\psi(M t+1)+\gamma_{0}} \leq P_{0} \leq \frac{P_{\max }}{\psi(M t+1)+\gamma_{0}}
$$

In the evaluation of the rate, we will consider the lower bound, giving a lower bound to the average rate of user $i$. The bounds for the power per stream derived by (30) with uniform power allocation are compared in Fig. 5 with the results obtained by simulations. We notice a very good agreement between

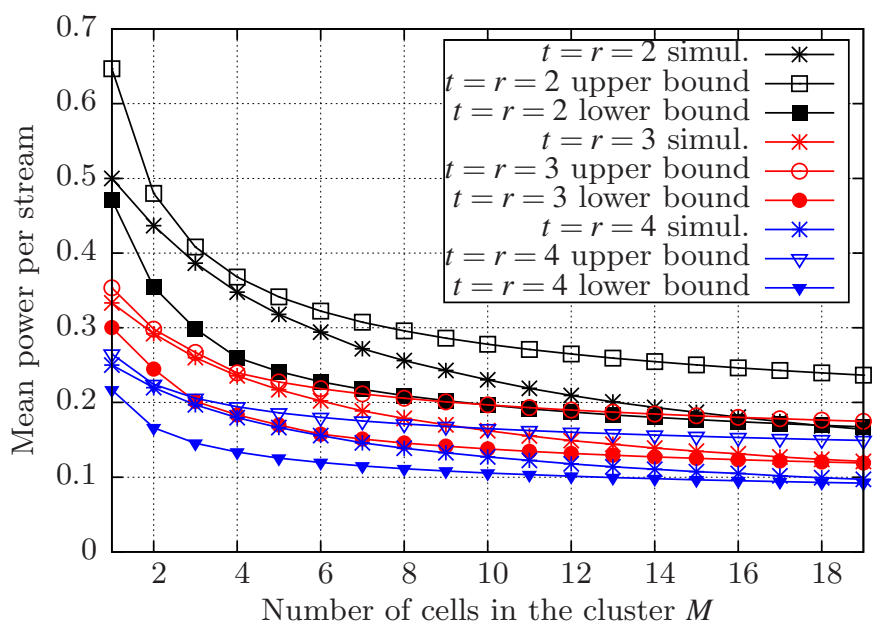

Fig. 5. Normalized average power per stream $P_{0} / P_{\max }$ for different antenna configurations: comparison between simulations and the bounds (29).

the analytical and simulation results, for different antenna configurations. In particular we see that for a small number of cells in the cluster the upper bound is quite tight, while, when the cluster size increases the lower bound provides a better approximation. This power decrease is affecting the mean achievable rate for an increasing size of the cluster, as discussed in the following results of Section V.

\section{Evaluation of the mean achievable rate}

The performance of the coordination scheme will be measured by the mean achievable rate per user in the cluster $\bar{R}$,

$$
\bar{R}=\frac{1}{N} \sum_{i=1}^{N} \overline{R_{i}}
$$

We can derive a lower bound for each user's average rate, by considering the inequality

$$
\log (1+\operatorname{SINR}) \geq \log (\operatorname{SINR}) .
$$

Then the integral (15) can be expressed as

$\overline{R_{i}} \simeq \frac{1}{\log 2} \sum_{\ell=1}^{r} \int_{0}^{R_{\text {cell }}} \log \left(\frac{P_{i \ell} \mu_{i \ell} u^{-\gamma}}{\sigma_{n}^{2}+P_{\max } M_{e q, i}\left(D_{i}-u\right)^{-\gamma}}\right) \frac{2 u}{R_{\text {cell }}^{2}} d u$,

where the model of interference has been introduced. Then

$$
\begin{aligned}
\overline{R_{i}} & \simeq \frac{1}{\log 2} \sum_{\ell=1}^{r}\left\{\log \left(\mu_{i \ell}\right)+\log \left(\frac{P_{i \ell}}{P_{\max }}\right)\right. \\
& \left.+\int_{0}^{R_{\text {cell }}} \log \left(\frac{u^{-\gamma}}{\frac{\sigma_{n}^{2}}{P_{\max }}+M_{\text {eq }, i}\left(D_{i}-u\right)^{-\gamma}}\right) \frac{2 u}{R_{\text {cell }}^{2}} d u\right\}
\end{aligned}
$$


We define $Z_{i}$ the integral part of (34), i.e.

$$
Z_{i}=\int_{0}^{R_{\text {cell }}} \log \left(\frac{u^{-\gamma}}{\frac{\sigma_{n}^{2}}{P_{\max }}+M_{e q, i}\left(D_{i}-u\right)^{-\gamma}}\right) \frac{2 u}{R_{\text {cell }}^{2}} d u .
$$

In the Appendix we detail the evaluation of $Z_{i}$, which gives

$$
\begin{aligned}
Z_{i} & =\frac{\gamma}{2}+\log (\rho)+d_{i}^{2} \log \left(\frac{d_{I}^{\gamma}}{M_{e q, i} \rho+d_{i}^{\gamma}}\right) \\
& +\frac{d_{i}^{2} \gamma}{2}\left[{ }_{2} \mathrm{~F}_{1}\left(1, \frac{2}{\gamma} ; \frac{\gamma+2}{\gamma} ; \frac{-d_{i}^{\gamma}}{M_{e q, i} \rho}\right)\right. \\
& \left.-4{ }_{2} \mathrm{~F}_{1}\left(1, \frac{1}{\gamma} ; \frac{\gamma+1}{\gamma} ; \frac{-d_{i}^{\gamma}}{M_{e q, i} \rho}\right)\right] \\
& +2 d_{I}\left(d_{i}-1\right) \gamma_{2} \mathrm{~F}_{1}\left(1, \frac{1}{\gamma} ; 1+\frac{1}{\gamma} ; \frac{-\left(d_{i}-1\right)^{\gamma}}{M_{e q, i} \rho}\right) \\
& -\frac{\left(d_{i}-1\right)^{2}}{2} \gamma_{2} \mathrm{~F}_{1}\left(1, \frac{2}{\gamma} ; 1+\frac{2}{\gamma} ; \frac{-\left(d_{i}-1\right)^{\gamma}}{M_{e q, i} \rho}\right) \\
& -\left(d_{i}^{2}-1\right) \log \left(\frac{\left(d_{i}-1\right)^{\gamma}}{M_{e q, i} \rho+\left(d_{i}-1\right)^{\gamma}}\right) .
\end{aligned}
$$

where ${ }_{2} \mathrm{~F}_{1}$ is the hypergeometric function and $\rho$ is the SNR defined in (2). Then, using (17) and (30), we obtain the analytical expression for the mean achievable rate per user

$$
\begin{aligned}
\bar{R} & =\frac{1}{\log 2}\left\{\frac{1}{N}\left[\sum_{\ell=1}^{N r} \psi(N r-\ell)+\log (F)\right]\right. \\
& \left.+r\left[\log \left(\frac{\Gamma(t+1)^{-1 / r}}{\psi(M t+1)+\gamma_{0}}\right)+\frac{\gamma}{2}+\log (\rho)\right]\right\} \\
& +\frac{1}{N \log 2} \sum_{i=1}^{N}\left\{d _ { i } ^ { 2 } \gamma \left[\frac{1}{2}{ }_{2} \mathrm{~F}_{1}\left(1, \frac{2}{\gamma} ; 1+\frac{2}{\gamma} ;-\frac{d_{i}^{\gamma}}{M_{e q, i} \rho}\right)\right.\right. \\
& \left.-2{ }_{2} \mathrm{~F}_{1}\left(1, \frac{1}{\gamma} ; 1+\frac{1}{\gamma} ;-\frac{d_{i}^{\gamma}}{M_{e q, i} \rho}\right)\right]+d_{i}^{2} \log \left(\frac{d_{i}^{\gamma}}{M_{e q, i} \rho+d_{I}^{\gamma}}\right) \\
& +2 d_{i}\left(d_{i}-1\right) \gamma_{2} \mathrm{~F}_{1}\left(1, \frac{1}{\gamma} ; 1+\frac{1}{\gamma} ;-\frac{\left(d_{i}-1\right)^{\gamma}}{M_{e q, i} \rho}\right) \\
& -\frac{1}{2}\left(d_{i}-1\right)^{2} \gamma_{2} \mathrm{~F}_{1}\left(1, \frac{2}{\gamma} ; 1+\frac{2}{\gamma} ;-\frac{\left(d_{i}-1\right)^{\gamma}}{M_{e q, i} \rho}\right) \\
& \left.-\left(d_{i}^{2}-1\right) \log \left(\frac{\left(d_{i}-1\right)^{\gamma}}{M_{e q, i} \rho+\left(d_{i}-1\right)^{\gamma}}\right)\right\},
\end{aligned}
$$

where $M_{e q, i}$ is given by (9) and $F$ by (20).

\section{NUMERICAL RESULTS}

We present results derived from the analytical expression (37) and from simulations in a scenario of 169 cells ( 7 concentric tiers of hexagonal cells), averaging 5,000 random trials. In each trial the positions of the users are randomly set according to a uniform distribution inside each cell as described in Section II and the channel is randomly generated according to the model of Section II-A. The parameter evaluated in the simulations is the achievable rate of (14), in which the values of powers, interference, the coefficients $\lambda_{i \ell}$, etc. are obtained by simulation. The clusters, despite being static, can have different shape for the same number of cells $M$. Indeed, in the simulations, the clusters are generated following an heuristic approach which tries to group cells in a compact way, with regular shape, by minimizing the sum of the inter-cells distances, thus to avoid long clusters, where the interfering cells from other clusters are closer. Note however that some values of the size $M$ do not allow for regular clusters (i.e. hexagonal) to be formed. If not otherwise stated, the following parameters are used: $\gamma=3.8$, cell radius $R_{\text {cell }}=1.4 \mathrm{~km}$ and $M=N$ with uniform distribution of the users within each cell.

\section{A. Comparison with simulations and with MMSE}

We first show a comparison between the expression (37), the simulation results, and an MMSE approach as in [11] for an antenna configuration $r=t=2$ and different values of the $\mathrm{SNR}$, defined as in (2). The results of BD refer to a uniform power allocation, so that a better rate could be obtained by power optimization, as shown in the following section. In Fig. 6 the mean rate is shown as a function of the cluster size $M$, showing that an optimal value of $M$ exists for BD. We show

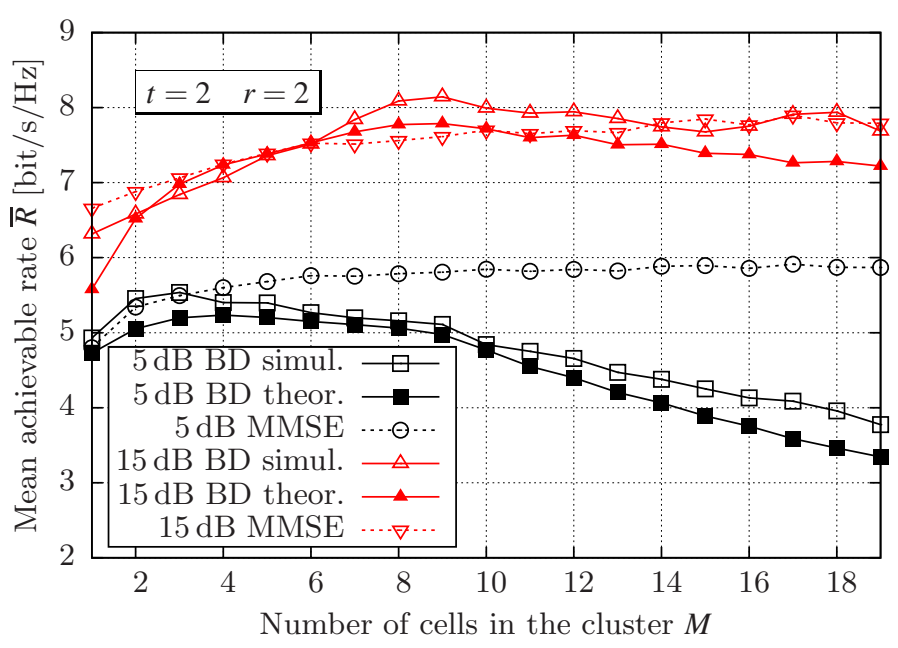

Fig. 6. Mean achievable rate per user as a function of the number of cells in the cluster for $r=2, t=2$, variable values of SNR, $\gamma=3.8$.

as expected that an MMSE approach can achieve better results in the case of low SNR, but even for moderate values of SNR, the BD approach gives comparable and even more favorable results, thus showing that the interference is dominating. We can see that even with MMSE the mean achievable rate tends to saturate with an increasing size $M$ of the clusters. The variability of the simulation results is not due to the limited number of trials, but to a natural variability of the rate with respect to the cluster shape, so that the level of interference does not change regularly with a change of the cluster size $M$, being some aggregations more favorable than others.

The same behavior pointed out in [12] is shown in Fig. 7 where saturation clearly appears after a certain value of SNR, depending on the propagation path-loss coefficient $\gamma$, which determines the influence of interference. In particular, the saturation SNR for BD is higher than for MMSE. For the first it is always above $20 \mathrm{~dB}$ for the considered scenarios, even for very small path loss coefficients. This means that the saturation occurs for relatively large SNR values, what is of practical importance. If we compare MMSE and BD under the restriction that the same $P_{\max }$ is transmitted for all $\gamma$, then the 


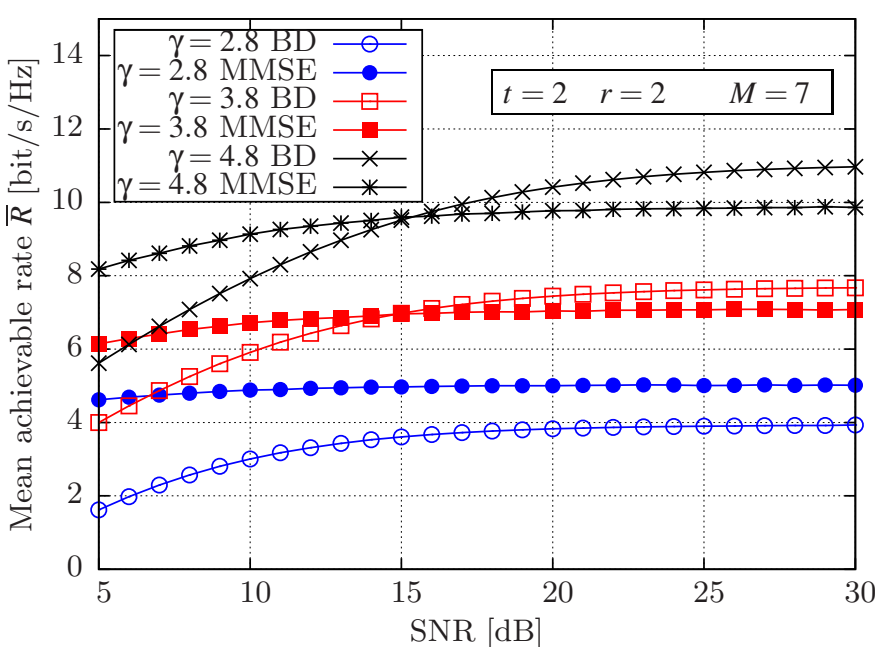

Fig. 7. Mean achievable rate as a function of the SNR for different values of the path-loss coefficient $\gamma$.

saturation occurs at different levels for each $\gamma$, although the general conclusions do not change.

To complete the validation of the theoretical results with the simulations, a fixed value of SNR and different numbers of antennas are considered in Fig. 8 and the good agreement between the theoretical and simulation results is still maintained, giving the same tendency, for $\mathrm{SNR}=25 \mathrm{~dB}$ and uniform power allocation. The small discrepancy between the

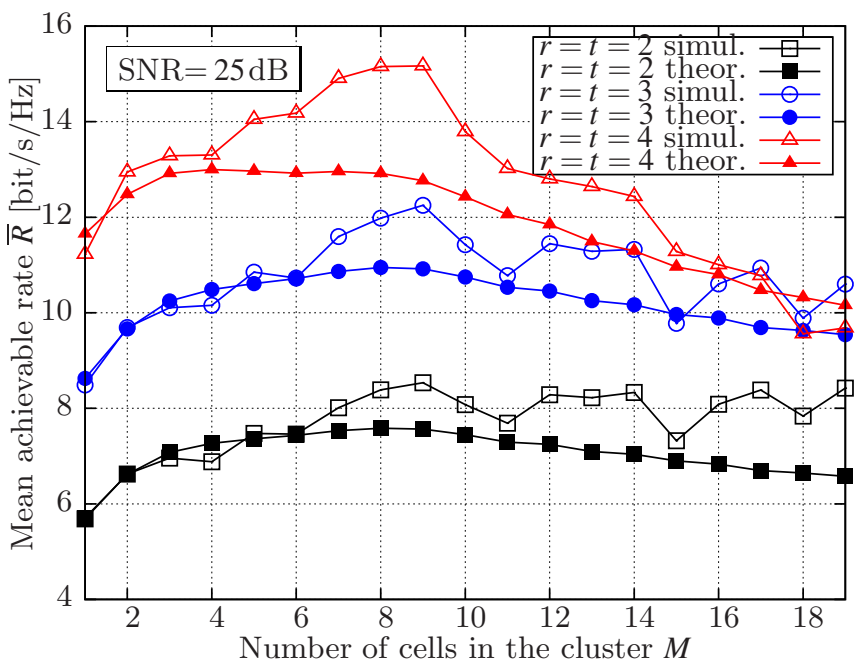

Fig. 8. Mean achievable rate per user as a function of the cluster size for $\mathrm{SNR}=25 \mathrm{~dB}, \gamma=3.8$ and different antenna configurations.

theoretical results and the simulations is not only due to the approximations, but also to the fact that in the simulation scenario not all the clusters have the same shape, despite having the same number of cells.

\section{B. Effect of the power allocation}

The decrease of the rate with respect of the cluster size is two-fold: on one hand we experience the decrease of the values $\lambda_{i \ell}$ and on the other hand, the power assigned to each stream decreases, due to a "coordination loss". If we assume a uniform power allocation the decrease is shown in Fig. 5. However, to remove the dependence of the rate on the power allocation scheme, we derived also the optimal power allocation, by numerical optimization, solving the convex problem of constrained optimization, as described also in [28].Then the optimal power is used in (37) instead of the value (30) corresponding to a uniform allocation. In Fig. 9 we compare the rate obtained with uniform and optimal power allocation, as a function of the cluster size $M$. We can see that, although

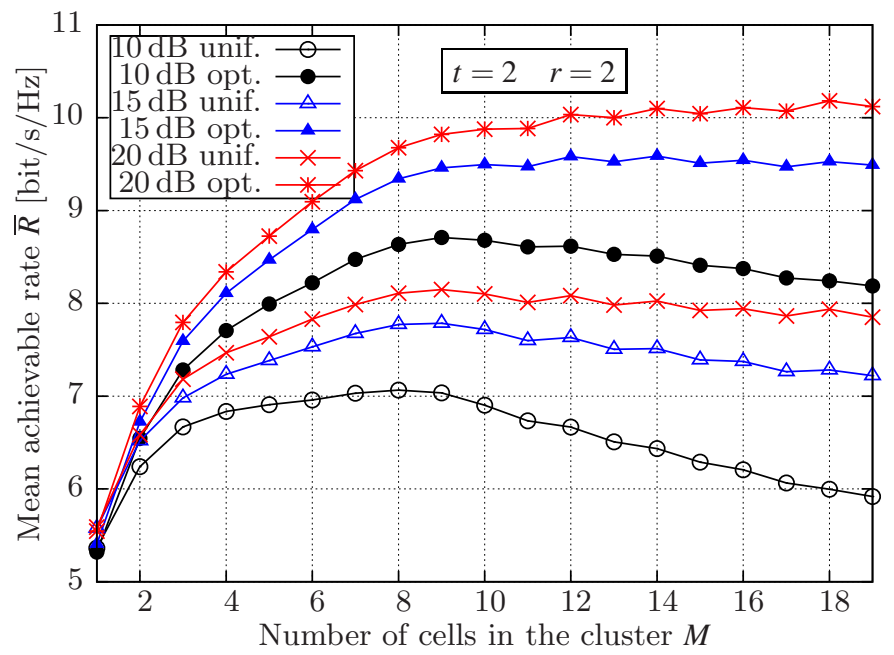

Fig. 9. Comparison between different power allocation schemes, namely uniform and optimal. Mean achievable rate per user as a function of the cluster size for $r=t=2, \gamma=3.8$ and different values of SNR.

the rates achieved by an optimal power allocation are higher, the curves follow a similar trend in both cases.

\section{Optimum cluster size}

From the results of Figs. 6-9 it is interesting to find the cluster size $M$ which can maximize the mean achievable rate. In Fig. 10 the optimum cluster size is plotted as a function of the SNR, for different antenna configurations and for the power allocation schemes considered. We can note that for a wide range of SNR the optimum value is limited, around 7-10 cells. Only for high SNR it is more convenient to increase the cluster size, since the reduction of interference can compensate the decrease of cluster-gain due to the decrease of the factors $\lambda_{i \ell}$. This occurs for greater values of $M$ if we consider the optimal power allocation, since in the case of uniform power we have an additional decrease of the rate, due to the reduction of the power per stream that can be allocated, as $M$ increases.

\section{Effect of signalling overhead}

In all the previous results the effect of the signalling overhead has not been taken into account, as in most of the published work [14]-[20]. However, if a certain percentage of the available resources are dedicated to channel estimation and signalling, the effective SNR and the payload are reduced with respect to the global achievable rate. In [12] the overhead, intended in that case solely for channel estimation purposes, 


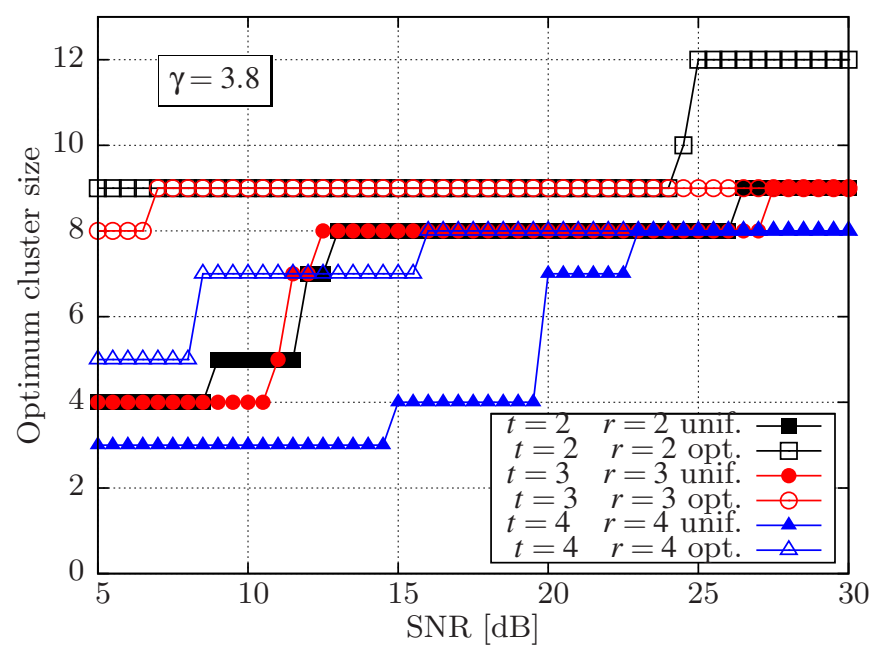

Fig. 10. Optimum cluster size as a function of the SNR, $\gamma=3.8$ and different antenna configurations.

is accouted for by a percentage $\alpha$, which should grow at least linearly with the cluster size. Then the effective SNR is $S N R_{e f f}=\operatorname{SNR}(1-\alpha)$ and the payload rate is $R_{e f f}=(1-\alpha) R$.

In order to show the effect of the overhead on the achievable rate, in Fig. 11 we adopt a very conservative approach in which the value of $\alpha$ grows only linearly with the cluster size $M$ up to the maximum value of $10 \%$ for $M=19$. We compare its effect, with the achievable rate obtained without overhead. Note that even with this small amount of overhead, increasing

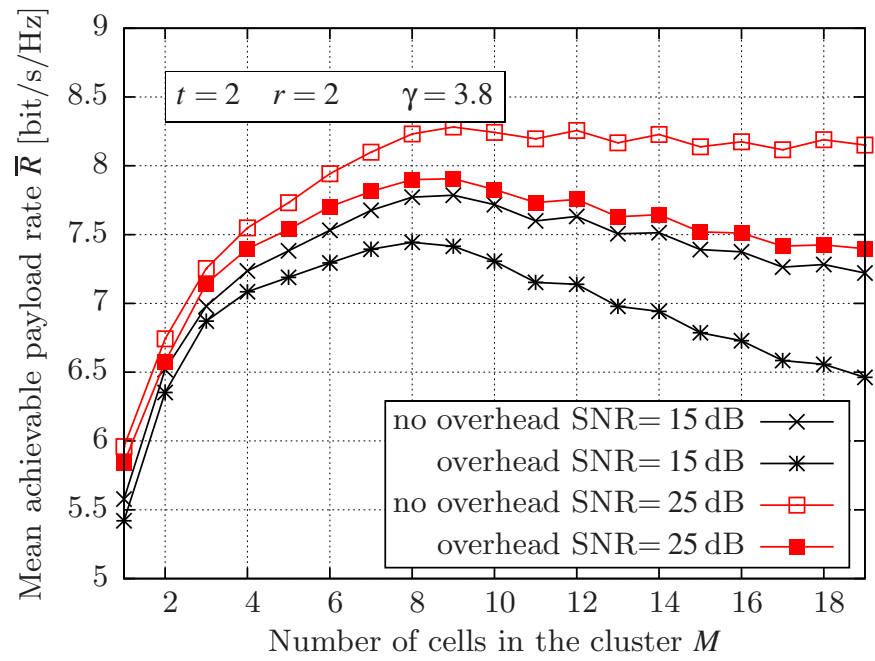

Fig. 11. Mean achievable rate and payload rate as a function of the cluster size $M$ with different SNRs.

values of $M$ lead to a worse performance. Moreover, we should remark that in general the actual definition of the signalling overhead and its management is delegated to the operator implementation, being hardly defined even in the standards. Thus its quantitative effect can change considerably depending on how the overhead is defined.

\section{CONCLUSIONS}

We studied a clustered base station coordination in the downlink transmission, using Block Diagonalization to remove interference among the users of the cluster. Since coordination is only performed within the cluster, users experience the interference from neighbor clusters, which depends on the cluster size. We derived an analytical expression for the mean achievable rate per user proposing an equivalent model for the interference, which allows to evaluate the impact of the main parameters, such as the SNR, the antenna configuration and the path-loss. These theoretical results can be used to derive higher order statistics or other performance parameters, as done in [32]. The main results can be thus summarized:

- In the analysis of the rate the actual position of the user is affecting more significantly the useful term of the power received from the nearest base station, while for the interference level and the power received from neighbor $\mathrm{BSs}$, the average position of the user in the cell provides a good approximation.

- Increasing the cluster size gives rise to a combination of effects, some with opposite consequences on the mean achievable rate: i) a reduction of the interference from the adjacent clusters, ii) a gain from coordination which soon saturates due to path-loss, and iii) a reduction of the power available for each stream in the case of uniform power allocation, due to the fewer degrees of freedom imposed by the BS power constraints.

- The maximum achievable rate can be obtained with a reduced cluster size (around 7-10) in a wide range of SNR values and for different antenna configurations.

- The same effect of saturation of the rate shown in [12] is confirmed. Fortunately, for BD it happens for relatively large SNR values, even in the case of small path loss coefficients with high interference conditions.

These conclusions apply to $\mathrm{BD}$ with fixed non-overlapping clusters, which is a possible and feasible way to perform the coordination in a clustered scenario. Although other methods such as MSSE or adaptive or user-centric clusters could give a better achievable rate, in general they require more complexity, at least in the management of the network.

\section{APPENDIX A}

DERIVATION OF $Z_{i}$

Normalizing the distance to the cell radius $R_{\text {cell }}$ the integral in (34) becomes

$$
\begin{aligned}
Z_{i} & =\int_{0}^{1} \log \left(\frac{u^{-\gamma}}{\frac{\sigma_{n}^{2}}{P_{\max } R_{c e l l}^{-\gamma}}+M_{e q, i}\left(d_{i}-u\right)^{-\gamma}}\right) 2 u d u \\
& =\int_{0}^{1} \log \left(\frac{u^{-\gamma}}{\frac{1}{\rho}+M_{e q, i}\left(d_{i}-u\right)^{-\gamma}}\right) 2 u d u \\
& =2 \int_{0}^{1} \log \left(\frac{1}{\frac{1}{\rho}+M_{e q, i}\left(d_{i}-u\right)^{-\gamma}}\right) u d u-2 \gamma \int_{0}^{1} \log (u) u d u
\end{aligned}
$$


where $\rho$ is the SNR of (2). The last integral in (38) is evaluated using 2.723 of [30], namely

$$
\int u \log (u) d u=u^{2}\left[\frac{\log u}{2}-\frac{1}{4}\right]
$$

and gives

$$
Z_{i}=\frac{\gamma}{2}+2 \int_{0}^{1} \log \left(\frac{1}{\frac{1}{\rho}+M_{e q, i}\left(d_{i}-u\right)^{-\gamma}}\right) u d u .
$$

The last integral in (40) is expressed as

$$
\begin{aligned}
& \int_{0}^{1} \log \left(\frac{1}{\frac{1}{\rho}+M_{e q, i}\left(d_{i}-u\right)^{-\gamma}}\right) u d u \\
& =\log (\rho)+\int_{d_{i}-1}^{d_{i}} \log \left(\frac{1}{1+M_{e q, i} \rho x^{-\gamma}}\right)\left(d_{i}-x\right) d x .
\end{aligned}
$$

The latter can be solved by the following result of [31],

$$
\begin{aligned}
& \int \log \left(\frac{1}{1+M_{e q, i} \rho x^{-\gamma}}\right)\left(d_{i}-x\right) d x \\
& =x\left[\frac{2 d_{i}-x}{2} \log \left(\frac{x^{\gamma}}{M_{e q, i} \rho+x^{\gamma}}\right)-d_{i} \gamma_{2} \mathrm{~F}_{1}\left(1, \frac{1}{\gamma} ; \frac{\gamma+1}{\gamma} ; \frac{-x^{\gamma}}{M_{e q, i} \rho}\right)\right. \\
& \left.\quad+\frac{x \gamma}{4}{ }_{2} \mathrm{~F}_{1}\left(1, \frac{2}{\gamma} ; \frac{\gamma+2}{\gamma} ; \frac{-x^{\gamma}}{M_{e q, i} \rho}\right)\right]
\end{aligned}
$$

where ${ }_{2} \mathrm{~F}_{1}$ is the hypergeometric function

$$
{ }_{2} \mathrm{~F}_{1}(a, b ; c ; z)=\frac{\Gamma(c)}{\Gamma(b) \Gamma(c-b)} \int_{0}^{1} \frac{x^{b-1}(1-x)^{c-b-1}}{(1-x z)^{a}} d x .
$$

Finally, we get

$$
\begin{aligned}
Z_{i} & =\frac{\gamma}{2}+\log (\rho)+d_{i}^{2} \log \left(\frac{d_{I}^{\gamma}}{M_{e q, i} \rho+d_{i}^{\gamma}}\right) \\
& +\frac{d_{i}^{2} \gamma}{2}\left[{ }_{2} \mathrm{~F}_{1}\left(1, \frac{2}{\gamma} ; \frac{\gamma+2}{\gamma} ; \frac{-d_{i}^{\gamma}}{M_{e q, i} \rho}\right)\right. \\
& \left.-4_{2} \mathrm{~F}_{1}\left(1, \frac{1}{\gamma} ; \frac{\gamma+1}{\gamma} ; \frac{-d_{i}^{\gamma}}{M_{e q, i} \rho}\right)\right] \\
& +2 d_{I}\left(d_{i}-1\right) \gamma_{2} \mathrm{~F}_{1}\left(1, \frac{1}{\gamma} ; 1+\frac{1}{\gamma} ; \frac{-\left(d_{i}-1\right)^{\gamma}}{M_{e q, i} \rho}\right) \\
& -\frac{\left(d_{i}-1\right)^{2}}{2} \gamma_{2} \mathrm{~F}_{1}\left(1, \frac{2}{\gamma} ; 1+\frac{2}{\gamma} ; \frac{-\left(d_{i}-1\right)^{\gamma}}{M_{e q, i} \rho}\right) \\
& -\left(d_{i}^{2}-1\right) \log \left(\frac{\left(d_{i}-1\right)^{\gamma}}{M_{e q, i} \rho+\left(d_{i}-1\right)^{\gamma}}\right) .
\end{aligned}
$$

\section{REFERENCES}

[1] M.K. Karakayali, G.J. Foschini, R.A. Valenzuela, "Network coordination for spectrally efficient communications in cellular systems," IEEE Wireless Comm., Vol. 13, No. 4, pp. 56-61, Aug. 2006.

[2] S. Shim, J.-S. Kwak, R.W. Heath, Jr., and J.G. Andrews, "Block Diagonalization for Multi-User MIMO with Other-Cell Interference", IEEE Trans. on Wireless Comm., Vol. 7, No. 7, pp. 2671-2681, July 2008.

[3] J.G. Andrews, Wan Choi, and R.W. Heath, "Overcoming Interference in Spatial Multiplexing MIMO Cellular Networks", IEEE Wireless Comm. Vol. 14, No. 6, pp. 95-104, Dec. 2007

[4] S. Shamai (Shitz) and B.M. Zaidel, "Enhancing the cellular downlink capacity via co-processing at the transmitting end," Proc. IEEE Vehicular Techn. Conference, Rhodes, Greece, May 2001.

[5] H. Zhang and H. Dai, "Cochannel Interference Mitigation and Cooperative Processing in Downlink Multicell Multiuser MIMO Networks", EURASIP Journal on Wireless Comm. and Networking, Vol. 2004, No. 2 pp. 222-235, Dec. 2004.
[6] Q.H. Spencer, A. L. Swindlehurst, and M. Haardt, "Zero-Forcing Methods for Downlink Spatial Multiplexing in Multiuser MIMO Channels", IEEE Trans. on Signal Processing, Vol. 52, No. 2, pp. 461-471, Feb. 2004.

[7] B.C. Lim, W.A. Krzymien and C. Schlegel, "Efficient Sum Rate Maximization and Resource Allocation in Block-Diagonalized SpaceDivision Multiplexing", IEEE Trans. on Vehic. Techn., Vol. 58, No. 1, pp. 478-484, Jan. 2009.

[8] J. Zhang, R. Chen, J.G. Andrews, A. Ghosh, and R.W. Heath, "Networked MIMO with Clustered Linear Precoding," IEEE Trans. on Wireless Comm., Vol. 8, No. 4, pp. 1910-1921, Apr. 2009.

[9] W. Liu, S.X Ng, and L. Hanzo, "Multicell Cooperation Based SVD Assisted Multi-User MIMO Transmission", Proc. IEEE Vehicular Techn. Conference, Barcelona, Spain, pp. 1-5, Apr. 2009.

[10] A. García Armada, R. Corvaja, M. Sánchez-Fernández, A. Santos Rodríguez, "MMSE Precoding for Downlink Coordinated Base Station Transmission", Proc. of IEEE Vehicular Techn. Conference, Budapest, Hungary, May. 2011.

[11] Q.J. Shi, M. Razaviyayn, C. He, and Z.-Q. Luo, "An Iteratively Weighted MMSE Approach to Distributed Sum-Utility Maximization for a MIMO Interfering Broadcast Channel", IEEE Trans. on Signal Processing, Vol. 59, pp. 4331-4340, March 2011.

[12] A. Lozano, R. Heath, and J. Andrews, "Fundamental limits of cooperation", arXiv:1204.0011, submitted to IEEE Trans. on Infomation Theory.

[13] A. Barbieri, P. Gaal, S. Geirhofer, T. Ji, D. Malladi, Y. Wei, and F. Xue, "Coordinated downlink multi-point communications in heterogeneous cellular networks", Proc. Inf. Theory and Applications Workshop (ITA), 2012.

[14] Huan Sun, Wei Fang and Lin Yang, "A Novel Precoder Design for Coordinated Multipoint Downlink Transmission", Proc. IEEE Vehicular Techn. Conference, Budapest, Hungary, May 2011.

[15] E. Björnson, R. Zakhour, D. Gesbert and B. Ottersten, "Cooperative Multicell Precoding: Rate Region Characterization and Distributed Strategies With Instantaneous and Statistical CSI", IEEE Trans. on Signal Processing, Aug. 2010.

[16] F. Boccardi and H. Huang, "Limited downlink network coordination in cellular networks," Proc. IEEE Int. Symp. on Personal, Indoor and Mobile Radio Communications (PIMRC '07), Athens, Greece, Sept. 2007.

[17] A. Papadogiannis, D. Gesbert, and E. Hardouin, "A dynamic clustering approach in wireless networks with multi-cell cooperative processing," Proc. IEEE Int. Conf. on Communications (ICC '08), Beijing, China, May 2008.

[18] Jung-Min Moon and Dong-Ho Cho, "Inter-cluster Interference Management based on Cell-clustering in Network MIMO Systems," Proc. IEEE Vehicular Techn. Conference, Budapest, Hungary, May 2011.

[19] E. Björnson, N. Jaldén, M. Bengtsson, and B. Ottersten, "Optimality Properties, Distributed Strategies, and Measurement-Based Evaluation of Coordinated Multicell OFDMA Transmission," IEEE Trans. on Signal Processing, vol. 59, pp. 6086-6101, Dec. 2011.

[20] H. Huang, M. Trivellato, A. Hottinen, M. Shafi, P. J. Smith and R. Valenzuela, "Increasing Downlink Cellular Throughput with Limited Network MIMO Coordination," IEEE Trans. on Wireless Comm., Vol. 8 No. 6, pp. 2983-2989, June 2009.

[21] Jun Zhang and J.G. Andrews, "Adaptive Spatial Intercell Interference Cancellation in Multicell Wireless Networks", IEEE Journal on Selected Areas in Communications, Vol. 28, No. 9, pp. 1455-1468, Dec. 2010.

[22] Jingya Li, C. Botella, and T. Svensson, "Resource allocation for clustered network MIMO OFDMA systems", EURASIP Journal on Wireless Comm. and Networking, DOI: 10.1186/1687-1499-2012-175.

[23] E. Björnson and E. Jorswieck, "Optimal resource allocation in coordinated multi-cell systems," Foundations and Trends in Communications and Information Theory, Vol. 9, No. 2-3, pp. 113-381, 2013.

[24] G. Caire, S. Shamai, "On the achievable throughput of a multiantenna Gaussian broadcast channel", IEEE Trans. on Information Theory, Vol. 49, No. 7, pp. 1691-1706, 2003.

[25] F. Boccardi, H. Huang, "A near-optimum technique using linear precoding for the MIMO broadcast channel", Proc. of IEEE Int. Conf. on Acoustics, Speech and Signal Processing, (ICASSP 2007), pp. III-17III-20, 2007.

[26] D. Ben Cheikh, J-M. Kelif, M. Coupechoux, P. Godlewski, "SIR distribution analysis in cellular networks considering the joint impact of path-loss, shadowing and fast fading", EURASIP Journal on Wireless Comm. and Networking, DOI: 10.1186/1687-1499-2011-137.

[27] B. Pijcke, M. Zwingelstein-Colin, M. Gazalet, M. Gharbi, P. Corlay, "An analytical model for the intercell interference power in the downlink of wireless cellular networks", EURASIP Journal on Wireless Comm. and Networking, DOI:10.1186/1687-1499-2011-95. 
[28] A. García Armada, M. Sánchez-Fernández, R. Corvaja, "Constrained Power Allocation Schemes for Coordinated Base Station Transmission using Block Diagonalization,", EURASIP Journal on Wireless Comm. and Networking, DOI: 10.1186/1687-1499-2011-125.

[29] A.M. Tulino, S. Verdù, "Random matrix theory and Wireless Comm.", Foundations and Trends in Communications and Information Theory, Vol. 1, No. 1, pp. 1-182, 2004.

[30] I.S. Gradshteyn, I.M. Ryzhik, Table of integrals, series, and products, Academic Press, 2000.

[31] Wolfram Alpha LLC. 2010. Wolfram-Alpha. www.wolframalpha.com.

[32] R. Corvaja, J.J. García Fernández, and A. García, "Partial Coordination in Clustered Base Station MIMO Transmission", Proc. IEEE WCNC 2013, Shanghai, China, Apr. 2013.

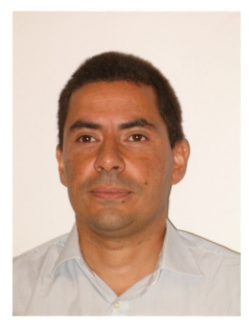

Roberto Corvaja Roberto Corvaja graduated in Electronic Engineering at the University of Padova (Italy) in 1990 and received the $\mathrm{PhD}$ in Electronic Engineering and Telecommunications at the University of Padova in 1994. Since 1994 he is with the Department of Information Engineering of the University of Padova as assistant professor in telecommunications. His research activity covered several aspects of digital communications, from coherent optical communications systems. to wireless communications and MIMO-OFDM wireless systems. In the last years his research included cooperative base station transmissions with precoding. He is co-author of two communications books and several journal papers. He served as TPC member of international conferences and as reviewer for several journals of IEEE.

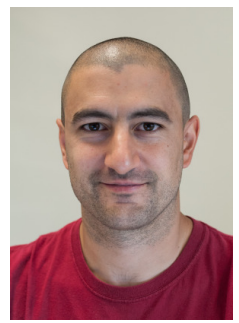

Juan José García Fernández Juan JosǴarcía Fernández received the M.S.E. in Electrical Engineering from the Universidad Carlos III de Madrid (Madrid, Spain), in 2009. He is currently working towards a Ph.D. degree at the Universidad Carlos III de Madrid. His current research focuses on cooperative approaches and energy efficiency for wireless communications.

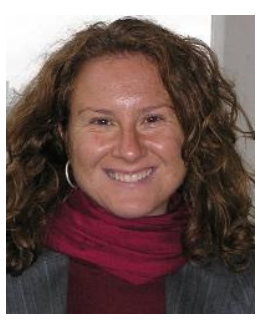

Ana García Armada Ana García Armada (S'96A'98-M'00-SM'08) received the Ph.D. degree in electrical engineering from the Polytechnic University of Madrid in February 1998. She is currently Professor at University Carlos III de Madrid, Spain, where she has occupied a variety of management positions. She is leading the Communications Research Group of this university and the Laboratory of Communication Systems for Security and Space in the Scientific Park of the same university. She has participated in several national and international research projects related to wireless communications. She is the coauthor of 8 book chapters on wireless communications and signal processing. She has published more than 35 papers in international journals and more than 50 contributions to international conference proceedings. She has contributed to international organizations such as ITU and ETSI. Her main interests are OFDM and MIMO techniques and signal processing applied to wireless communications. 